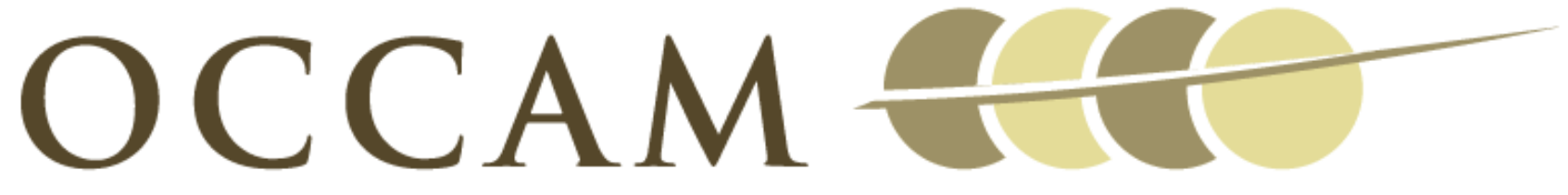

OXFORD CENTRE FOR COLLABORATIVE APPLIED MATHEMATICS

Report Number 11/26

Synaptic bistability due to nucleation and evaporation of receptor clusters

by

V. M. Burlakov, N. Emptage, A. Goriely and P. C. Bressloff

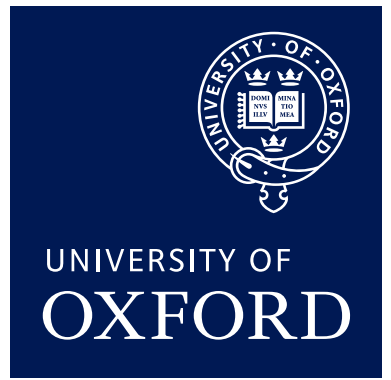

Oxford Centre for Collaborative Applied Mathematics Mathematical Institute 24 - 29 St Giles'

Oxford

OX1 3LB

England 



\title{
Synaptic bistability due to nucleation and evaporation of receptor clusters
}

\author{
V. M. Burlakov ${ }^{1}$, N. Emptage ${ }^{2}$, A. Goriely ${ }^{1}$ and P. C. Bressloff ${ }^{3}$ \\ ${ }^{1}$ Mathematical Institute, OCCAM, University of Oxford, 24-26 St Giles, Oxford OX1 3LB,UK \\ ${ }^{2}$ Department of Pharmacology, University of Oxford, Mansfield Road, Oxford OX1 3QT, UK \\ ${ }^{3}$ Department of Mathematics, University of Utah, Salt Lake City, UT 84112
}

\begin{abstract}
We introduce a bistable mechanism for long-term synaptic plasticity based on switching between two metastable states that contain significantly different numbers of synaptic receptors. One state is characterized by a two-dimensional gas of mobile interacting receptors and is stabilized against clustering by a high nucleation barrier. The other state contains a receptor gas in equilibrium with a large cluster of immobile receptors, which is stabilized from growing further by the turnover rate of receptors into and out of the synapse. Transitions between the two states can be initiated by either an increase (potentiation) or a decrease (depotentiation) of the net receptor flux into the synapse. This changes the saturation level of the receptor gas and triggers nucleation or evaporation of receptor clusters.
\end{abstract}

PACS numbers: 87.19.lw,87.19.lv,87.19.lg

According to current understanding, the main biophysical mechanism for storing information in the central nervous system is activity-based changes in the strengths or efficacies of synaptic connections between neurons (synaptic plasticity) [1-4]. In many cases, synaptic plasticity is expressed by changes in the number of neurotransmitter protein receptors within the postsynaptic membrane of a stimulated neuron $[5,6]$. However any modification in the number of independent receptors cannot fully account for long-term memory because of the limited dwell time of individual receptors, which are recycled into and out of a synapse several times an hour [7]. One suggested mechanism for stabilizing higher concentrations of receptors at a synapse is through interactions with submembrane scaffolding proteins [8-10]. An alternative mechanism has recently been proposed by Shouval [11], in which receptor clusters can survive much longer than individual receptors if the rate of receptor insertion into the membrane (exocytosis) is higher in the vicinity of other receptors due to receptor-receptor interactions and the rate of receptor removal from the membrane (endocytosis) is independent of such interactions. However, no particular mechanism for cluster formation has previously been proposed.

In this Letter we present a physical model of receptor stabilization that includes an explicit mechanism for receptor clustering. The latter is formulated along analogous lines to the well-known phenomenon of vapor-liquid phase transformations in supercritical liquid droplet nucleation and growth [12]. Vapour in our model is represented by mobile receptors within the postsynaptic membrane. We show that supercritical clusters can quickly be nucleated at a pre-existing nucleation site (heterogeneous nucleation [13]) if the surrounding vapor becomes dense enough, e.g. after an excitatory signal has stimulated the synapse. The nucleation site can be generated by any receptor that has been localized by interactions with sub-membrane scaffolding proteins. Once nucleated, the cluster grows until it reaches an equilibrium size determined by the balance between the rates of endocytosis and exocytosis. The cluster can also be evaporated if the receptor concentration in the vapor becomes too low, e.g. after an inhibitory signal. We show that such a mechanism of cluster formation/evaporation allows a synapse to exhibit bistability, i.e. there exist two distinct stable steady states with different efficacies (receptor numbers). Receptors in the state with low efficacy are predominantly in the vapor phase, while the efficacy of the other state is significantly increased by the formation of one or more receptor clusters. Transitions between the two stable states correspond to writing or erasing information. We also show that synaptic bistability is controlled by the total influx of receptors into the postsynaptic terminal and depends on the area of the postsynaptic membrane. Changing any of these parameters modifies the size and stability of the receptor cluster and modulates synaptic efficacy accordingly.

Model description. At most excitatory synapses in the brain, receptors are highly clustered at the postsynaptic density (PSD), which is the protein-rich domain in the postsynaptic membrane of a dendritic spine (see Fig. 1). The dendritic spine is a small (sub-micrometer) membranous extrusion that protrudes from a dendrite. Typically spines have a bulbous head that is connected to the parent dendrite through a thin spine neck. There are two main mechanisms for the transport of receptors between the PSD and extrasynaptic regions of the spine and dendrite [9]. First, surface receptors can undergo lateral membrane diffusion, in which the PSD acts as a spatially localized trap through receptor interactions with scaffolding proteins and the cytoskeleton. This is consistent with single-particle tracking experiments, which show surface receptors undergoing periods of free diffusion interspersed with periods of restricted motion in confinement domains that coincide with synapses [14-17]. Second, surface receptors may be internalized via endo- 
cytosis and either stored within an intracellular pool or recycled to the surface via exocytosis [18].

In our model of receptor clustering we will focus on the second transport process and neglect lateral diffusion between the PSD and the extrasynaptic membrane (see Fig. 1). Thus, we assume that exocytosis and endocytosis oc-

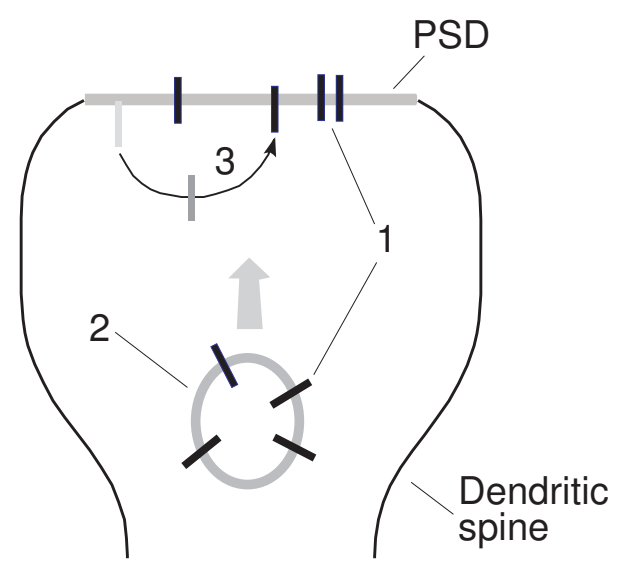

FIG. 1: Schematic representation of the PSD within a dendritic spine. The number of receptors ( 1 ) within the PSD is maintained by receptor recycling (3). Extra receptors can be delivered to the PSD from an intracellular pool (2) following an excitatory signal to form metastable clusters, which results in potentiation (strengthening) of the synapse. An inhibitory signal would cause an increase in the rate of receptor removal (endocytosis), resulting in receptor cluster evaporation and weakening of the synapse (depotentiation).

cur directly in the PSD [19]. We also assume that surface receptors within the PSD are either mobile or part of a stationary cluster. At any time $t$ the total number of receptors of the PSD will depend on the balance between fluxes into and out of the postsynaptic membrane. The total receptor influx has two components $y=y_{1}+y_{2}$, where $y_{1}$ is the influx due to local receptor recycling and $y_{2}$ is the contribution from an intracellular receptor pool triggered upon synaptic excitation (see Fig. 1). The outflow of receptors depends on the numbers of clustered and mobile receptors, with endocytosis rates determined by their local environments. One candidate mechanism for receptor clustering is short-range (Van der Waals) interactions between receptors with a characteristic energy of the order $k_{B} T$, where $T$ is room temperature [20]. This relatively low binding energy allows us to treat receptor clusters as two-dimensional liquid droplets, which are approximately circular as they minimize their surface energy. Hence, any receptor removal from the cluster does not change the cluster shape and integrity.

Energy analysis. Although receptor diffusion within the PSD is 10-50 times slower than within the extrasynaptic membrane [14-17], it is still fast enough to establish thermodynamic equilibrium of the receptor vapor. This allows us to define the chemical potential of recep- tors in the cluster $\mu_{\mathrm{cl}}$ and in the vapor $\mu_{\mathrm{vap}}$ as

$$
\begin{aligned}
\mu_{\mathrm{cl}} & =-\varepsilon_{\mathrm{cl}}-\varepsilon_{\mathrm{adh}}+\gamma \frac{a}{R}, \\
\mu_{\mathrm{vap}} & =k_{B} T \ln \left(x_{\mathrm{vap}} \Lambda^{2}\right)-\varepsilon_{\mathrm{adh}},
\end{aligned}
$$

where $\varepsilon_{\mathrm{cl}}$ is the binding energy of receptors in the cluster, $R$ is the cluster radius, $\varepsilon_{\text {adh }}$ is the adhesive energy of receptors in the membrane, $\gamma$ is the cluster surface energy per receptor, $a$ is the spacing between receptors in the cluster, and $\Lambda$ is the receptor thermal wavelength in the vapor [21]. If we explicitly modeled receptor diffusion using a lattice gas model (see Ref. [22]) then the thermal wave length would be equal to $a$.

The vapor concentration $x_{\text {vap }}$ can be obtained by equating the receptor influx $y$ with the receptor outflow at steady state, and assuming that the area $S_{0}$ occupied by the mobile receptors (vapor) is approximately independent of the size of the cluster. The outflow of receptors contains two contributions: one from the clustered receptors and the other from the mobile ones. The balance of receptor fluxes in and out of the PSD is

$$
y=\pi R^{2} \nu \mathrm{e}^{-\varepsilon_{\mathrm{cl}}-\varepsilon_{\text {adh }}+\gamma / R}+S_{0} x_{\mathrm{vap}} \nu \mathrm{e}^{-\varepsilon_{\text {adh }}},
$$

where, for convenience, we express all lengths in $a$-units and all energies in $k_{B} T$-units. The product $P_{\text {vap }}=$ $\nu \mathrm{e}^{-\varepsilon_{\text {adh }}}$ is the endocytosis rate of receptors in the vapor, with $\nu$ the attempt frequency (taken to be the same for both mobile and bound receptors). Similarly, the product $P_{\mathrm{cl}}=\nu \mathrm{e}^{-\varepsilon_{\mathrm{adh}}-\varepsilon_{\mathrm{cl}}+\gamma / R}$ is the endocytosis rate from the cluster, which assumes that the interaction between receptors naturally increases the dwell time for bound synaptic receptors (see also [14]) in accordance with their binding energy $\varepsilon_{\mathrm{cl}}$. Note that this is entirely opposite to the approach used in Ref. [11], where receptor removal rates were assumed to be the same for mobile and bound receptors. Rearranging Eq. (2) we obtain the vapor concentration as a function of cluster size:

$$
x_{\mathrm{vap}}(R)=\frac{y}{S_{0} P_{\mathrm{vap}}}-\frac{\pi R^{2}}{S_{0}} \mathrm{e}^{-\varepsilon_{\mathrm{cl}}+\gamma / R} .
$$

In the limit $R \rightarrow 1$ (in $a$-units), we can neglect the second term on the RHS of Eq. (3) so that $x_{\mathrm{vap}}(0)=$ $y /\left(S_{0} P_{\text {vap }}\right)$. Using this expression and writing the cluster radius $R=R\left(n_{\mathrm{cl}}\right) \equiv \sqrt{n_{\mathrm{cl}} / \pi}$ in terms of receptor number in the cluster $n_{\mathrm{cl}}$ we obtain the energy required for formation of the receptor cluster from the vapor:

$$
\begin{aligned}
\Delta E= & \int_{0}^{n_{\mathrm{cl}}}\left(\mu_{\mathrm{cl}}-\mu_{\mathrm{vap}}\right) d n+S_{0}\left[F\left(n_{\mathrm{cl}}\right)-F(0)\right] \\
= & -n_{\mathrm{cl}} \varepsilon_{\mathrm{cl}}-\int_{0}^{n_{\mathrm{cl}}} \ln \left[x_{\mathrm{vap}}(0)-\frac{n}{S_{0}} \mathrm{e}^{-\varepsilon_{\mathrm{cl}}+\gamma \sqrt{\pi / n}}\right] d n \\
& \quad+2 \gamma \sqrt{\pi n_{\mathrm{cl}}}+S_{0}\left[F\left(n_{\mathrm{cl}}\right)-F(0)\right]
\end{aligned}
$$

where $F(n)=x_{\text {vap }}(R(n)) \ln \left(x_{\text {vap }}(R(n))\right.$. Fig. 2 shows the dependence of the system energy upon the cluster 


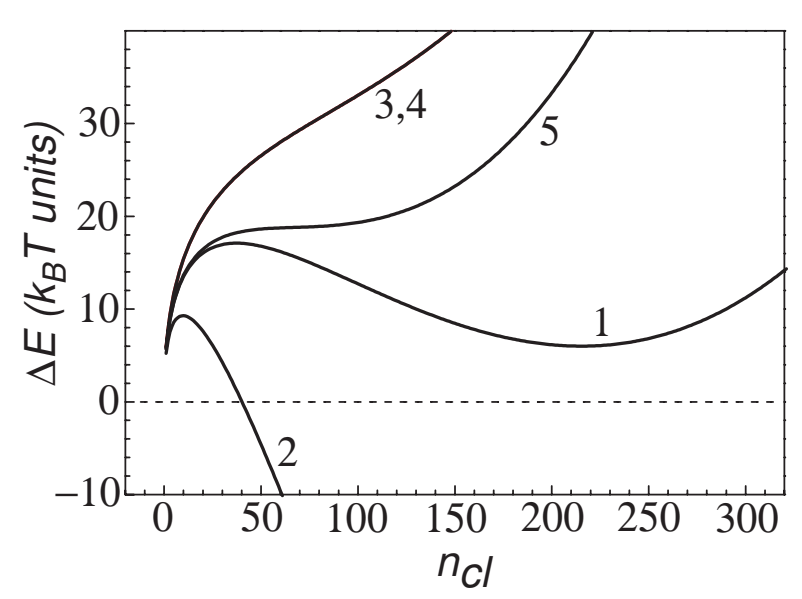

FIG. 2: The energy change associated with cluster formation as a function of cluster number $n_{\mathrm{cl}}$. The energy was obtained by evaluating Eq. (4) for $\varepsilon_{\mathrm{cl}}=5, \gamma=1.646$ and 1) $P_{\mathrm{vap}}=$ $\left.\left.0.1, S_{0}=500, y=0.6 ; 2\right) P_{\text {vap }}=0.1, S_{0}=500, y=0.9 ; 3\right)$ $P_{\text {vap }}=0.1, S_{0}=500, y=0.5$; 4) $P_{\text {vap }}=0.12, S_{0}=500, y=$ 0.6 ; 5) $P_{\text {vap }}=0.1, S_{0}=300, y=0.36$. The latter value of $y$ is chosen to keep $x_{\mathrm{vap}}(0)$ the same as in 1 .

size for different values of the receptor influx $y$, endocytosis rate $P_{\text {vap }}$ and synaptic area $S_{0}$. The value of $\gamma$ is chosen in order to describe correctly the surface energy of relatively small discrete cluster of receptors on a hexagonal grid (see section 1 in Supporting Material). Also note that the value $P_{\text {vap }}=0.1 \mathrm{~min}^{-1}$ corresponds to a dwell time of extrasynaptic receptors given by $t_{d}=10 \mathrm{~min}$ [15]. For $y=0.6$ (curve 1) the energy as a function of cluster size $n_{\mathrm{cl}}$ has two local minima. The one at $n_{\mathrm{cl}}=0$ corresponds to the state with no cluster, while the other corresponds to a state with a cluster of size $n_{\mathrm{cl}} \approx 200$. To nucleate this cluster, the system has to overcome an energy barrier of about $\delta E \approx 17 k_{B} T$ in height and about $\delta n \approx 50$ receptors in width, which makes spontaneous cluster formation rather unlikely. If the overall receptor influx is increased up to $y=0.9$ (curve 2) by activating an intracellular pool, for example, the energy of the clustered state rapidly decreases, making cluster formation very likely, as both the nucleation barrier height and the width are significantly decreased down to $\delta E \approx 10 k_{B} T$ and $\delta n \approx 15$, respectively. This suggests that an increase in $y$ for a relatively short time period may be enough to initiate cluster formation by appropriate fluctuations. The cluster may then grow further and reach its stable size even after the influx $y$ has returned to its background value $y=y_{1}$. The reverse phenomenon of cluster evaporation takes place if the influx $y$ is decreased or the endocytosis rate $P_{\text {vap }}$ is increased, as illustrated by the coincident curves 3 and 4 . An important feature of the proposed mechanism is illustrated by curves 1 and 5 in Fig. 2, which show that increasing the synaptic area $S_{0}$ whilst keeping the influx density $y / S_{0}$ fixed results in an increased and more stable synaptic efficacy. This behavior is consistent with experimental data suggesting that increasing dendritic spine size (hence $S_{0}$ ) stabilizes a potentiated synapse [23, 24].

Model kinetics. To study transitions between the two metastable states of receptor number, we consider the following equations governing the time evolution of the populations of clustered $n_{\mathrm{cl}}$ and mobile $N_{\text {vap }}$ receptors:

$$
\begin{aligned}
\frac{d n_{\mathrm{cl}}}{d t} & =2 \sqrt{\pi n_{\mathrm{cl}}}\left(J_{\mathrm{con}}-J_{\text {evap }}\right)-n_{\mathrm{cl}} P_{\mathrm{cl}} \\
\frac{d N_{\mathrm{vap}}}{d t} & =y-N_{\text {vap }} P_{\text {vap }}-2 \sqrt{\pi n_{\mathrm{cl}}}\left(J_{\text {con }}-J_{\text {evap }}\right)-n_{\mathrm{cl}} P_{\mathrm{cl}}
\end{aligned}
$$

where $J_{\text {con }}$ and $J_{\text {evap }}$ are, respectively, the fluxes of condensing and evaporating receptors from the cluster. We take $J_{\text {con }}=\beta x_{\text {vap }} D / 4$, where $D /(4 \lambda)$ is the rate at which receptors collide with the cluster surface, and $\beta$ is the probability for these receptors to be incorporated into the cluster. Here $D$ is the diffusion coefficient of mobile receptors in the PSD, and $\lambda$ is the receptor mean free path. For hopping-like diffusion in a lattice-gas model, $\lambda$ can be taken equal to $a$. The factor $1 / 4$ takes into account the fraction of receptors moving towards the cluster surface. We have made an implicit assumption that the receptor vapor is in thermodynamic equilibrium, i.e. homogeneous, which is well justified if $\beta \ll 1$. The flux $J_{\text {evap }}=\beta x_{\mathrm{GT}} D / 4$ can be obtained by assuming that the cluster evaporates with the same rate as if it was in equilibrium with the surrounding vapor, and its stability is due to the compensating condensation flux from the vapor. The so-called Gibbs-Thomson concentration $x_{\mathrm{GT}}$ of the vapor can be found from the equilibrium condition $\mu_{\mathrm{cl}}=\mu_{\mathrm{vap}}$ with the chemical potentials given by Eqs (1). Hence, $x_{\mathrm{GT}}=\mathrm{e}^{-\varepsilon_{\mathrm{cl}}+\gamma \sqrt{\pi / n_{\mathrm{cl}}}}$.

We solved Eqs. (5) using kinetic Monte Carlo simulations (see section 2 of Supporting Material and Ref. [25]). Typical simulation results illustrating responses to an increased receptor influx (potentiation) or receptor removal rate (depotentiation) are presented in Fig. 3 . In the simulations we used $a=5 n m, \beta=0.005$ and a characteristic diffusion coefficient $D$ for mobile receptors in the PSD [14-17]. We also assumed that the PSD contains a single nucleation site with at least two receptors. The impact of the parameter $\beta D$ on the cluster size and its fluctuations is described in section 3 of the Supporting Material. We find that our simulation results are in excellent agreement with the energy analysis if $\beta D>80 a^{2} / s$. The values of other parameters used for the simulations shown in Fig. 3a are the same as those used to calculate curve 1 in Fig. 2. Cluster formation was triggered by a short increase in $y_{2}$ from 0 to 1.2 , after which the cluster grows to its stable size of around 200 receptors, and fluctuates about this stable value. To initiate cluster evaporation we increased $P_{\text {vap }}$ by factor of 3. The results shown in Fig. 3b were obtained using parameter values for curve 5 in Fig. 2. Increasing $y_{2}$ 


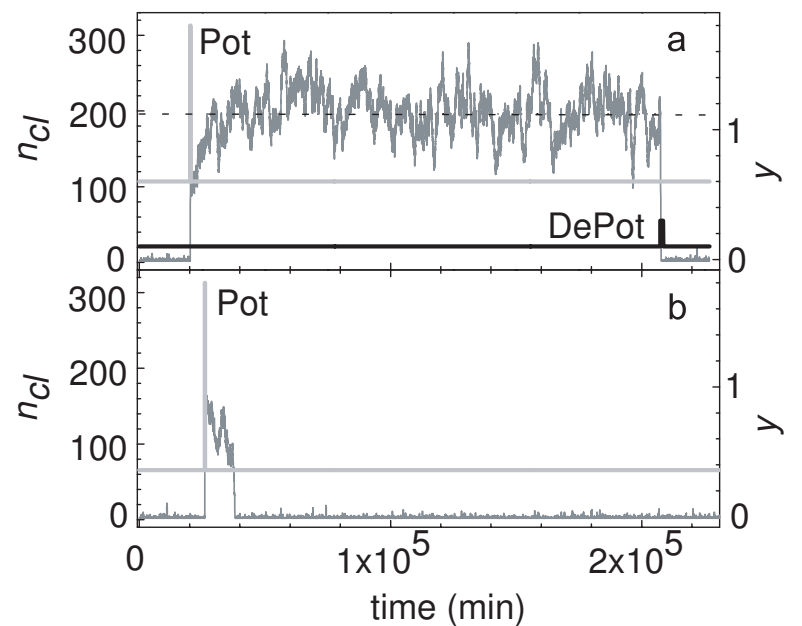

FIG. 3: Evolution of the cluster receptor number $n_{\mathrm{cl}}$ (dark gray curve) obtained by kinetic Monte Carlo simulations of Eq. (5) with $P_{\text {vap }}=0.1, \varepsilon_{\mathrm{cl}}=5, \gamma=1.646, D=$ $10^{4} \mathrm{~nm}^{2} / \mathrm{s}, \beta=0.005$. a) $S_{0}=500, y_{1}=0.6$; (b) $S_{0}=$ $300, y_{1}=0.36$. Light gray line shows total receptor influx $y=y_{1}+y_{2}$, where $y_{1}$ represents the background exocytosis rate due to receptor recycling and $y_{2}$ represents the spike in receptor influx from an intracellular pool due to potentiation (Pot). In (a) dotted line indicates the steady state cluster size and the black line indicates the spike in the endocytosis rate corresponding to a depotentiation (DePot) signal.

now induces the formation of an unstable cluster, which decays spontaneously after about 150 hours.

Discussion. In this Letter we presented a robust, biophysica model of synaptic bistability based on the nucleation or evaporation of receptor clusters around existing nucleation sites in the postsynaptic membrane. Bistability is controlled by the area of the synapse and the background receptor vapor concentration. Both metastable states of the synapse can be maintained with the same recycling rate of receptors, and hence at the same metabolic cost to the cell. We found that the long-term stability (lifetime) of synaptic efficacy is achieved with the formation of large (200 receptors) clusters while smaller clusters may account for the shorter term potentiation of synaptic efficacy. One major simplification of the model was to consider only a single cluster. In our simulations we found that nucleation of more than one cluster at different nucleation sites does not change the main result related to synaptic efficacy, as only one stable cluster is eventually formed and all the others disappear. This result also addresses another implicit assumption regarding non-clustered immobile receptors: they do not contribute to the vapor-cluster dynamics and need only be treated as additional nucleation sites. A more detailed study, however, is required to investigate the impact of multiple nucleation sites on fluctuations in receptor number.

Acknowledgments: This work was funded by the
John Fell Oxford University Press (OUP) Research Fund. In part, it was also supported by King Abdullah University of Science and Technology Award No. KUK-C1013-04. AG and PCB are Wolfson/Royal Society Merit Award holders. NE thanks the Medical Research Council (UK) for support.

[1] S. J. Martin, P. D. Grimwood and R. G. M. Morris, Annu. Rev. Neurosci. 23, 649 (2000).

[2] R. Malinow and R. C. Malenka, Annu. Rev. Neurosci. 25, 103 (2002).

[3] M. Sheng and M. J. Kim, Science 298, 776 (2002).

[4] D. S. Bredt and R. A. Nicoll, Neuron 40, 361 (2003).

[5] S. H. Shi, Y. Hayashi, R. S. Petralia, et al., Science 284, 1811 (1999).

[6] Y. Hayashi, S. H. Shi, J. A. Esteban, et al., Science 287, $2262(2000)$.

[7] F. Crick, Nature 312, 101 (1984).

[8] J. E. Lisman and M. A. Goldring, Proc. Nat. Acad. Sci. USA 85, 5320 (1988).

[9] Triller and D. Choquet, Trends Neurosci. 28, 133 (2005).

[10] K. Sekimoto and A. Triller, Phys. Rev. E 79, 031905 (2009).

[11] H. Z. Shouval, Proc. Nat. Acad. Sci. USA 85, 14440 (2005).

[12] J. Frenkel, Kinetic Theory of Liquids (Oxford University Press, Oxford, 1946); P. G. Debenedetti, Metastable Liquids (Princeton University Press, Princeton, 1996).

[13] Laaksonen, V. Talenquer and D. W. Oxtoby, Ann. Rev. Phys. Chem. 46, 489 (1995).

[14] H. Adesnik, R. A. Nicoll and P. M. England, Neuron 48, 977 (2005).

[15] E. M. Petrini, J. Lu, L. Cognet, et al., Neuron 63, 92 (2009).

[16] C. Tardin, L. Cognet, C. Bats, B. Lounis and D. Choquet, EMBO J 22, 4656 (2003).

[17] V. M. Burlakov, R. Taylor, J. Koerner and N. Emptage, Biophys. J. 99, 1368 (2010).

[18] J-H Tao-Cheng et al. J. Neurosci. 31, 4834 (2011)

[19] There is strong experimental evidence that insertion and removal of surface receptors occurs at extrasynaptic sites so that lateral diffusion is required for receptors to enter or exit the PSD [18]. For simplicity, we lump these processes together. However, it would be straightforward to extend the model to include the effects of surface diffusion between the PSD and extrasynatic sites.

[20] M. A. Wear, D. Kan, A. Rabu and M. D. Walkinshaw, Angew. Chem. Int. Ed. 46, 6453 (2007).

[21] Z. Yan, Eur. J. Phys. 21, 625 (2000).

[22] J. W. Evans, D-J. Liu and M. Tammaro, Chaos 12, 131 (2002).

[23] E. A. Nimchinsky, B. L. Sabatini and K. Svoboda, Annu. Rev. Physiol. 64, 313 (2002).

[24] H. Kasai, M. Matsuzaki, J. Noguchi, et al., Trends Neurosci. 26, 360 (2003).

[25] K. A. Fichthorn and W. H. Weinberg, J. Chem. Phys. 95(2), 1090 (1991). 



\section{RECENT REPORTS}

03/11 Spontaneous rotational inversion in Phycomyces Goriely

Tabor

04/11 From individual to collective behaviour of coupled velocity jump Erban processes: a locust example

Haskovec

05/11 Solving Eigenvalue problems on curved surfaces using the closest MacDonald point method

Brandman

Ruuth

06/11 A numerical methodology for the Painleve equations

Fornberg

Weideman

07/11 Strong stability preserving two-step Runke-Kutta methods

Ketcheson

Gotlieb

MacDonald

08/11 Hysteresis and Post Walrasian Economics

Cross

McNamara

Kalachev

Pokrovskii

09/11 A locally adaptive time-stepping algorithm for petroleum reservoir simulations

McNamara

Bowen

Dellar

10/11 On the predictions and limitations of the BeckerDoring model for reaction kinetics in micellar surfactant solutions

Griffiths

Bain

Breward

Colegate

Howell

Waters

11/11 Dynamics of the Tear Film

Braun

12/11 The infuence of receptor-mediated interactions on reactiondiffusion mechanisms of cellular self-organisation

Klikaa

Baker

Headon

Gaffney

13/11 Quasi-steady state analysis of two-dimensional random intermittent search processes

Bressloff

Newby

14/11 A Constrained Approach to Multiscale Stochastic Simulation of Chemically Reacting Systems

Cotter

Zygalakis

Kevrekidis

Erban

15/11 The Two Regime Method for optimizing stochastic reactiondiffusion simulations

Flegg

Chapman

Erban

16/11 Recombination via tail states in polythiophene:fullerene solar cells

Kirchartz

Pieters

Kirkpatrick

Rau

Nelson

17/11 Energy versus electron transfer in organic solar cells: a comparison Soon 
19/11 A perturbation analysis of spontaneous action potential initiation by stochastic ion channels

Keener1

Newby

20/11 Hybrid modelling of individual movement and collective behaviour

Franz

Erban

21/11 A novel model for one-dimensional morphoelasticity. Part I: Theoretical foundations

Hall

Menon

$\mathrm{McCue}$

McElwain

22/11 A novel model for one-dimensional morphoelasticity. Part II: Ap-

Hall plication to the contraction of fibroblast-populated collagen lattices

Menon

$\mathrm{McCue}$

McElwain

23/11 Positive or negative Poynting efect? The role of adscititious in-

Mihai equalities in hyperelastic materials

Goriely

McCue

McElwain

24/11 On approaches to modelling lattice dislocations

Hall

Markenscoff

25/11 Nonlinear waves in heterogeneous elastic rods via homogenization

de Luna

Duričković

Goriely

Copies of these, and any other OCCAM reports can be obtained from:

Oxford Centre for Collaborative Applied Mathematics Mathematical Institute

24 - 29 St Giles'

Oxford

OX1 3LB

England

www.maths.ox.ac.uk/occam 